\title{
„Ungehobener Schatz": Die Chancen von Big Data
}

Irgendwo zwischen dem Heiligen Gral, dem Stein der Weisen und dem Schatz im Silbersee liegt der Stellenwert, den Big Data in der Zukunft einnehmen wird. In diese Richtung zumindest ging die Diskussion um „Das Potenzial von Big Data für Forschung und Medizin“, bei welcher der Raum beim Hauptstadtkongress bis auf den letzten Platz gefüllt war.

Es sind diese riesigen Datenmengen, die unentwegt von Patienten erhoben werden und zumindest bisher nicht systematisch, gebündelt und über Algorithmen ausgewertet werden, die Forscher, Informatiker und Mediziner gleichermaßen faszinieren. „Die großen Datenmengen sind ein großer Schatz, der noch nicht gehoben wurde“, sagte der Parlamentarische Staatssekretär im Bundesministerium für Bildung und Forschung (BMBF), Thomas Rachel (CDU). Die gezielte Auswertung und der Einsatz von Big Data könne die Gesundheitsforschung verbessern und „,ist ein wichtiger Schritt für den medizinischen Fortschritt“, zeigte sich Rachel überzeugt.

\section{Zielgerichtete Auswertung für Innovation}

„Professionell, standardisiert aufbereitet und intelligent verknüpft bergen diese Daten ein großes Potenzial für bessere Diagnostik und Therapie." Das BMBF legt dafür ein 100-Millionen-Euro-Förderprogramm für Universitätskliniken auf, um ein deutschlandweit vernetztes IT-Infrastruktursystem zu unterstützen. Aspekte des Datenschutzes, rechtliche und ethische Fragen müssten einbezogen werden, betonte Rachel, damit es gelingt, die „skeptischen Teilnehmer am Gesundheitswesen“ von den Vorteilen des Teilens von Daten und ihrer Auswertung zu überzeugen. Andere Länder seien bereits deutlich weiter, was ein vernetztes Gesundheitssystem angehe. Ziel sei die bestmögliche Versorgung von Patienten durch die Entwicklung indivi-

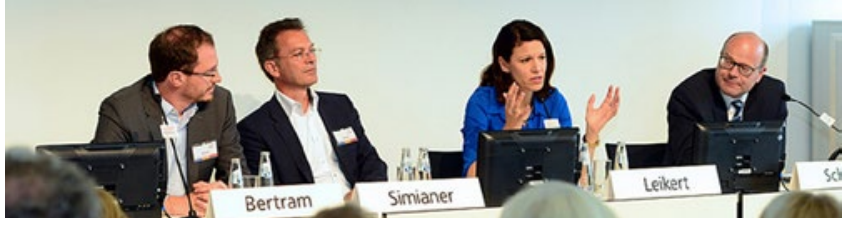

Big Data bleibt eine große Herausforderung für die Zukunft.

dualisierter Medizin - ohne die Preisgabe personenbezogener Daten. Nur durch das Zusammenführen von Daten und der zielgerichteten Auswertung sei Innovation möglich. Fragen zum Datenschutz und dem Eigentum von erhobenen Forschungsdaten (Daten-Ownership) aus dem Publikum blieben jedoch weitgehend unbeantwortet.

Oliver Schenk, Leiter der Abteilung Grundsatzfragen der Gesundheitspolitik und Telematik beim Bundesministerium für Gesundheit, wies den Vorwurf, Deutschland trage in Sachen Digitalisierung die rote Laterne, weit von sich. „Wir wären gern woanders als da, wo wir heute stehen“, sagte er. „Aber wir sind auf einem guten Weg.“ Dr. Katja Leikert (CDU), Mitglied im Gesundheitsausschuss des Bundestages, verwies in diesem Zusammenhang auf „diverse Verzögerungsprozesse“, seit 2004 die Einführung der elektronischen Gesundheitskarte beschlossen worden sei. „Wenn nur theoretisch über Digitalisierung und Datenaustausch gesprochen wird, dann gibt es viele Vorbehalte“, sagte sie. Die Vorteile, vor allem für die Patienten, würden weniger wahrgenommen. Inzwischen allerdings sei - nicht zuletzt wegen des E-Health-Gesetzes - eine „neue Dynamik“auch für Big Data entstanden.

\section{Unterschiedliche Vorstellungen zur Finanzierung des Gesundheitswesens}

Ihre Konzepte zur zukünftigen Finanzierung des Gesundheitswesens haben die gesundheitspolitischen Sprecherinnen von CDU/CSU, SPD und Bündnis 90/Die Grünen in der Bundestagsrunde vorgestellt. Dabei wurde deutlich, dass vor allem die Koalitionsparteien bei der Parität in der gesetzlichen Krankenversicherung (GKV) meilenweit auseinander liegen.

„Wir werden noch in dieser Legislaturperiode die Chance ergreifen, die Festschreibung der Arbeitgeberbeiträge zur Krankenversicherung zu beenden“, versprach die gesundheitspolitische Sprecherin der SPD-Bundestagsfraktion Hilde Mattheis. Aus ihrer Sicht wird mit der Rückkehr zur paritätischen Finanzierung durch Arbeitgeber und Arbeitnehmer die „erste Stellschraube für eine Finanzierungssolidarität“ in der GKV gedreht. Gleichzeitig nehme die hälftige Finanzierung der Krankenversicherungsbeiträge den Druck bei den womöglich weiter steigenden Zusatzbeiträgen heraus. Für eine Rückkehr zur Parität plädierte auch die Grünen-Sprecherin Maria Klein-Schmeink. „Der Zusatzbeitrag ist ein Irrweg, denn die Solidarität wird damit nur weiter ausgehöhlt“, sagte sie und kritisierte, dass der Zu-

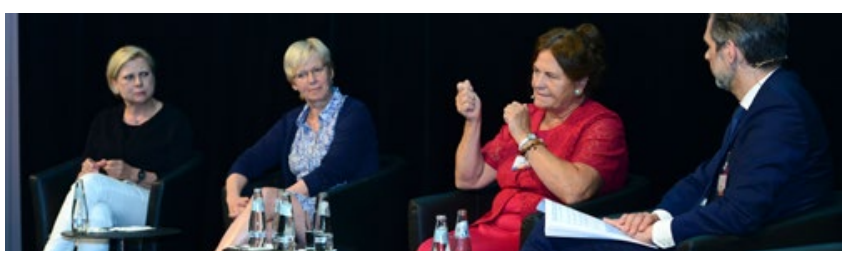

Parität oder Nicht-Parität: drei Sprecherinnen, zwei Meinungen.

satzbeitrag einen Preiswettbewerb zwischen den Kassen hervorrufe und diese davon abhalte, die Versorgung zu sichern.

Die Sprecherin der Unionsfraktion für Gesundheitsfragen, Maria Michalk, verteidigte den festgeschriebenen GKV-Arbeitgeberanteil auf 7,3 Prozent vehement. „Wir halten aus ordnungspolitischen Gründen daran fest“, erklärte Michalk. „Was in wirtschaftlich schlechten Zeiten gut war, muss auch in wirtschaftlich guten Zeiten nicht schlecht sein“. Auch der von SPD und Grünen geforderten Bürgerversicherung erteilte die CDUPolitikerin erneut eine klare Absage: „Die Bürgerversicherung ist kein Rezept für die Zukunft.“ 